PROCEEDINGS OF THE

AMERICAN MATHEMATICAL SOCIETY

Volume 125, Number 5, May 1997, Pages 1561-1570

S 0002-9939(97)03971-3

\title{
ON THE STABILITY OF EQUIVARIANT FOLIATIONS
}

\author{
JANEZ MRČUN
}

(Communicated by Ronald Stern)

\begin{abstract}
We prove an equivariant version of the Reeb-Thurston stability theorem for foliations invariant under an action of a discrete group.
\end{abstract}

Let $G$ be a discrete group and $\mu: M \times G \rightarrow M$ a right $C^{1}$-action of $G$ on a connected $C^{1}$-manifold $M$ of dimension $n$ without boundary. Let $\mathcal{F}$ be a $C^{1}$ foliation of codimension $k$ on $M$ which is invariant under the action of $G$, i.e. for any $g \in G$ the diffeomorphism $\hat{g}=\mu(-, g)$ of $M$ maps leaves into leaves. We will write $\mu(x, g)=x \cdot g$.

If $L$ is a leaf of $\mathcal{F}$, the isotropy group of $L$ is the subgroup of $G$

$$
G_{L}=\{g \in G \mid L \cdot g \subset L\} .
$$

The action of $G$ restricts to a $C^{1}$-action of $G_{L}$ on $L$. The immersion of $L$ into $M$ induces a continuous injection of the orbit space $L / G_{L}$ into the orbit space $M / G$. If $L^{\prime}$ is another leaf of $\mathcal{F}$, the orbit spaces $L / G_{L}$ and $L^{\prime} / G_{L^{\prime}}$ are either disjoint or isomorphic over $M / G$, so $\mathcal{F}$ induces a partition of the space $M / G$. If the action of $G$ is free and properly discontinuous, the orbit space $M / G$ is a $C^{1}$-manifold and the induced partition is the induced $C^{1}$-foliation $\mathcal{F} / G$ on $M / G$. In general, the induced partition of $M / G$ may be seen as a generalized foliation.

The aim of this paper is to prove a version of the Reeb-Thurston stability theorem $[11,12]$ for such a generalized foliation. But before we can state the theorem, we have to formalize the intuitive notion of the transversal part of the action of $G$.

Let $L$ be a leaf of $\mathcal{F}$ and $x_{0} \in L$. Denote by $\pi_{1}^{G}\left(L ; x_{0}\right)$ the set of pairs $(g, \sigma)$ with $g \in G_{L}$ and $\sigma$ a homotopy class (with fixed end-points) of a path in $L$ which starts at $x_{0}$ and ends at $x_{0} \cdot g$. Define an operation in $\pi_{1}^{G}\left(L ; x_{0}\right)$ by

$$
\left(g^{\prime}, \sigma^{\prime}\right)(g, \sigma)=\left(g^{\prime} g, \hat{g}_{*}\left(\sigma^{\prime}\right) \sigma\right),
$$

where $\hat{g}_{*}\left(\sigma^{\prime}\right) \sigma$ is the concatenation of the homotopy classes $\sigma$ and $\hat{g}_{*}\left(\sigma^{\prime}\right)$. With this operation $\pi_{1}^{G}\left(L ; x_{0}\right)$ becomes a group, called the equivariant fundamental group of $L$. There is a canonical inclusion $i n c_{L}: \pi_{1}\left(L ; x_{0}\right) \rightarrow \pi_{1}^{G}\left(L ; x_{0}\right)$ given by $i n c_{L}(\sigma)=$ $(1, \sigma)$, and a canonical projection $p r_{L}: \pi_{1}^{G}\left(L ; x_{0}\right) \rightarrow G_{L}$ given by $\operatorname{pr}_{L}(g, \sigma)=g$. The sequence of homomorphisms of groups

$$
1 \longrightarrow \pi_{1}\left(L ; x_{0}\right) \stackrel{i n c_{L}}{\longrightarrow} \pi_{1}^{G}\left(L ; x_{0}\right) \stackrel{p r_{L}}{\longrightarrow} G_{L} \longrightarrow 1
$$

Received by the editors December 1, 1995.

1991 Mathematics Subject Classification. Primary 57R30, 57S25.

Key words and phrases. Foliation, group action.

This work was partially supported by the Ministry of Science of Slovenia.

(C)1997 American Mathematical Society 
is exact. Note that if $x_{0}^{\prime}$ is another point of $L$, then a path in $L$ between $x_{0}$ and $x_{0}^{\prime}$ induces an isomorphism $\pi_{1}^{G}\left(L ; x_{0}\right) \cong \pi_{1}^{G}\left(L ; x_{0}^{\prime}\right)$. Therefore we will write $\pi_{1}^{G}(L)$ for the isomorphism class of the equivariant fundamental group of $L$.

Note that $\pi_{1}^{G}(L)$ is naturally isomorphic to the fundamental group of the Borel construction $L \times_{G_{L}} \mathbf{E} G_{L}$, where $\mathbf{E} G_{L}$ is a universal $G_{L}$-bundle. This follows from the facts that the action of $G_{L}$ on $L \times \mathbf{E} G_{L}$ is free and properly discontinuous and that $\mathbf{E} G_{L}$ is contractible. In particular, if $G_{L}$ acts freely and properly discontinuously on $L$, then the covering projection $L \rightarrow L / G_{L}$ induces an isomorphism $\pi_{1}^{G}(L) \cong \pi_{1}\left(L / G_{L}\right)$.

Choose a transversal section $T: \mathbf{R}^{k} \rightarrow M$ of $(M, \mathcal{F})$ with $T(0)=x_{0}$, and let $(g, \sigma)$ be an element of $\pi_{1}^{G}\left(L ; x_{0}\right)$. Now $\hat{g} \circ T$ is a transversal section with $(\hat{g} \circ T)(0)=$ $x_{0} \cdot g$. The holonomy of $\sigma$ relative to the sections $T$ and $\hat{g}_{\circ} T$ defines a germ $\operatorname{Hol}_{\hat{g} \circ T, T}(\sigma)$, which is an element of the group $\operatorname{Diff}_{0}\left(\mathbf{R}^{k}\right)$ of germs at $0 \in \mathbf{R}^{k}$ of diffeomorphisms $f$ between open neighbourhoods of 0 in $\mathbf{R}^{k}$ with $f(0)=0$. We define the equivariant holonomy homomorphism of the leaf $L$ with respect to the transversal section $T$

$$
\operatorname{Hol}_{T}^{G}: \pi_{1}^{G}(L ; T(0)) \longrightarrow \operatorname{Diff}_{0}\left(\mathbf{R}^{k}\right)
$$

by $\operatorname{Hol}_{T}^{G}(g, \sigma)=\operatorname{Hol}_{\hat{g} \circ T, T}(\sigma)$. Note that the restriction of $\operatorname{Hol}_{T}^{G}$ on the fundamental group $\pi_{1}(L ; T(0))$ is the usual holonomy homomorphism $\mathrm{Hol}_{T}$ of $L$ with respect to $T$. If $T^{\prime}$ is another transversal section with $T^{\prime}(0) \in L$ and $\psi: \pi_{1}^{G}(L ; T(0)) \rightarrow$ $\pi_{1}^{G}\left(L ; T^{\prime}(0)\right)$ is the isomorphism induced by a path in $L$ between $T(0)$ and $T^{\prime}(0)$, then the homomorphisms $\mathrm{Hol}_{T}^{G}$ and $\mathrm{Hol}_{T^{\prime}}^{G} \circ \psi$ differ by the conjugation by a canonically determined element of $\operatorname{Diff}_{0}\left(\mathbf{R}^{k}\right)$, i.e. we have a canonical isomorphism $\mathrm{Hol}_{T}^{G} \cong \mathrm{Hol}_{T^{\prime}}^{G}$ 。$\psi$. Therefore we will write

$$
\mathrm{Hol}_{L}^{G}: \pi_{1}^{G}(L) \longrightarrow \operatorname{Diff}_{0}\left(\mathbf{R}^{k}\right)
$$

for the isomorphism class of the equivariant holonomy homomorphism of $L$. The group $\operatorname{Hol}_{L}^{G}\left(\pi_{1}^{G}(L)\right)$ is called the equivariant holonomy group of $L$.

Remark. Intuitively, for any $g \in G_{L}$ the germ $\operatorname{Hol}_{T}^{G}(g, \sigma)$ is the transversal part of the diffeomorphism $\hat{g}$ around $L$, but determined up to the choice of a homotopy class $\sigma$ of a path from $x_{0}$ to $x_{0} \cdot g$. In fact, it is only the holonomy class of $\sigma$ which matters. The geometric picture is particularly clear if the group $G_{L}$ has a fixed point in $L$, so we can choose $x_{0}$ to be that fixed point. In this case there is a splitting

$$
s: G_{L} \longrightarrow \pi_{1}^{G}\left(L ; x_{0}\right)
$$

of the sequence (1) given by $s(g)=(g, 1)$, and $\pi_{1}^{G}\left(L ; x_{0}\right)$ is a semi-direct product of $G_{L}$ and $\pi_{1}\left(L ; x_{0}\right)$. In particular, the transversal part of $\hat{g}$ may be seen as the germ $\operatorname{Hol}_{T}^{G}(s(g))$. In general, since $\pi_{1}\left(L ; x_{0}\right)$ is a normal subgroup of $\pi_{1}^{G}\left(L ; x_{0}\right)$, it follows that the holonomy group $\operatorname{Hol}_{T}\left(\pi_{1}\left(L ; x_{0}\right)\right)$ of $L$ is a normal subgroup of the equivariant holonomy group $\operatorname{Hol}_{T}^{G}\left(\pi_{1}^{G}\left(L ; x_{0}\right)\right)$ of $L$. The quotient

$$
\operatorname{Hol}_{T}^{G}\left(\pi_{1}^{G}\left(L ; x_{0}\right)\right) / \operatorname{Hol}_{T}\left(\pi_{1}\left(L ; x_{0}\right)\right)
$$

is thus the contribution of the action of $G$ to the equivariant holonomy of $L$.

The subgroup

$$
G_{L}^{\sharp}=p r_{L}\left(\operatorname{Ker~Hol}_{L}^{G}\right)
$$


of $G_{L}$ does not depend on the choice of a transversal section through the leaf $L$. An element $g \in G_{L}$ belongs to $G_{L}^{\sharp}$ if and only if there exists a homotopy class $\sigma$ of a path in $L$ from a point $x$ to $x \cdot g$ such that

$$
\operatorname{Hol}_{L}^{G}(g, \sigma)=1 .
$$

With $x$ fixed, the class $\sigma$ is determined uniquely up to holonomy. According to the previous remark, $G_{L}^{\sharp}$ may be regarded as the subgroup of those elements of $G_{L}$ which act transversely trivially around $L$.

The important fact about the group $G_{L}^{\sharp}$ is that it canonically acts on the holonomy covering space of the leaf $L$, i.e. on a covering space of $L$ which corresponds to the kernel of the holonomy homomorphism of $L$. Explicitly, if $\chi: L^{\sharp} \rightarrow L$ is a holonomy covering projection, we define for any $g \in G_{L}^{\sharp}$ and $y \in L^{\sharp}$

$$
y \cdot g=\tilde{\varphi}(1),
$$

where $\varphi$ is a path in $L$ with $\varphi(0)=\chi(y), \varphi(1)=\varphi(0) \cdot g$ such that $\operatorname{Hol}_{L}^{G}(g,[\varphi])=1$, and $\tilde{\varphi}$ is the unique lift of $\varphi$ in $L^{\sharp}$ with $\tilde{\varphi}(0)=y$. Here $[\varphi]$ denotes the homotopy class of the path $\varphi$. Since such a path is uniquely determined up to holonomy, the definition makes sense. It gives a right $C^{1}$-action of $G_{L}^{\sharp}$ on $L^{\sharp}$ such that $\chi$ is equivariant. Hence $\chi$ induces a continuous map $\chi^{G}: L^{\sharp} / G_{L}^{\sharp} \rightarrow L / G_{L}$.

We say that the action of $G$ is leafwise separated if the orbit space $L^{\sharp} / G_{L}^{\sharp}$ is Hausdorff, for any leaf $L$ of $\mathcal{F}$.

The action of $G$ is equivariantly transversely orientable around a leaf $L$ of $\mathcal{F}$ if all the germs in $\operatorname{Hol}_{L}^{G}\left(\pi_{1}^{G}(L)\right)$ preserve the orientation of $\mathbf{R}^{k}$. Note that if $\mathcal{F}$ is transversely orientable and $g \in G_{L}$ then all the germs in $\operatorname{Hol}_{L}^{G}\left(\operatorname{pr}_{L}^{-1}(g)\right)$ either preserve or invert the orientation of $\mathbf{R}^{k}$.

Example 1. (1) Let $p: M \rightarrow N$ be a submersion between connected (not necessarily Hausdorff) $C^{1}$-manifolds without boundary, and let $\mathcal{F}$ be the foliation on $M$ induced by $p$. Further assume that we have a $C^{1}$-action of a discrete group $G$ on $M$ such that

$$
p(x \cdot g)=p(x)
$$

for any $x \in M$ and $g \in G$. Since the leaves of $\mathcal{F}$ are the connected components of the fibers of $p$, the foliation $\mathcal{F}$ is invariant under the action of $G$. If $L$ is a leaf of $\mathcal{F}$, it is clear that both the holonomy group and also the equivariant holonomy group of $L$ are trivial. Thus $L^{\sharp} \cong L$ and $G_{L}^{\sharp}=G_{L}$. In particular, the action of $G$ is leafwise separated if and only if the orbit spaces of the leaves of $\mathcal{F}$ are Hausdorff.

Denote by $q: M \rightarrow M / G$ the quotient projection. The submersion $p$ induces a continuous map $p_{G}: M / G \rightarrow N$ with $p=p_{G} \circ q$. Let $y \in p(M)$. The restriction

$$
\left.q\right|_{p^{-1}(y)}: p^{-1}(y) \longrightarrow p_{G}^{-1}(y)
$$

is an open surjection. Since $p$ is a submersion, the fiber $p^{-1}(y)$ is locally connected, and hence so is the fiber $p_{G}^{-1}(y)$. This implies that if $L$ is a leaf of $\mathcal{F}$ then $q(L)$ is a connected component of the corresponding fiber of $p_{G}$. Moreover, the restriction $\left.q\right|_{L}: L \rightarrow q(L)$ is open, hence $q(L) \cong L / G_{L}$. In other words, the orbit spaces of the leaves of $\mathcal{F}$ are isomorphic with the connected components of the fibers of $p_{G}$.

(2) Let $M=\mathbf{R}^{2} \backslash\{0\}$ and let $\mathcal{F}$ be given by the submersion $p=p r_{2}: M \rightarrow \mathbf{R}$. Take $G=\mathbf{Z}$ and define a properly discontinuous free action of $G$ on $M$ by

$$
(x, y) \cdot g=\left(\mathrm{e}^{g} x, \mathrm{e}^{g} y\right)
$$


for any $(x, y) \in M$ and $g \in G$. The foliation $\mathcal{F}$ is invariant under this action. The induced foliation $\mathcal{F} / G$ on the orbit space $M / G \cong T^{2}$ has exactly two compact leaves. Any other leaf of $\mathcal{F} / G$ has both the compact leaves in its closure.

Any leaf of $\mathcal{F}$ has trivial fundamental group and trivial holonomy. Also, any leaf $L$ of $\mathcal{F}$ with $0 \notin p(L)$ has trivial isotropy group, therefore trivial equivariant fundamental group and trivial equivariant holonomy group. Now let $L$ be the leaf $\{(x, 0) \mid x>0\}$ of $\mathcal{F}$. The isotropy group of $L$ is clearly all $G$, and also the equivariant fundamental group and the equivariant holonomy group of $L$ are isomorphic with $\mathbf{Z}$.

This example shows that we cannot expect a stability theorem without assuming that the equivariant holonomy group of a leaf with compact orbit space is finite.

Let us now state our first theorem which expresses an equivariant version of the Reeb stability [11]:

Theorem 2. Let $\mathcal{F}$ be a $C^{1}$-foliation on a connected $C^{1}$-manifold $M$ without boundary, invariant under a leafwise separated $C^{1}$-action of a discrete group $G$ on $M$. Let $L$ be a leaf of $\mathcal{F}$ such that

(i) the orbit space of $L$ is compact, and

(ii) the equivariant holonomy group of $L$ is finite.

Then for any $G$-saturated open neighbourhood of $L$ there exists a smaller $G$-saturated open neighbourhood of $L$ which is a union of leaves of $\mathcal{F}$ with compact orbit spaces.

Remark. In the case $G=1$ this theorem expresses the simplest version of the Reeb stability theorem: a compact leaf with finite holonomy group has a neighbourhood of compact leaves. The Reeb stability theorem also says that the foliation around a compact leaf $L$ with finite holonomy group is that of a foliated bundle with the holonomy group of $L$ as the structure group. However, the equivariant analog of this fact is not true: see Example 6 (1).

For the proof, one may try to use a suspension $M \times_{G} \Omega$, where $\Omega$ would be a manifold with a properly discontinuous free $G$-action. If the equivariant holonomy group of a leaf $L$ of $\mathcal{F}$ is finite, the holonomy group of the corresponding leaf of the foliation on $M \times_{G} \Omega$ would be finite. Yet, the corresponding leaf would in general not be compact, so one cannot apply the classical Reeb stability theorem to this case. Instead, we shall rather use the principal bundle associated to $\mathcal{F}$. The starting point of the proof is an observation that the action of $G$ on $M$ lifts to an action on the principal bundle such that the bundle becomes equivariant. With this, we will be able to reduce the proof to the special case where the foliation is given by a submersion as in Example 1 (1). We shall thus first prove the theorem for this special case:

Lemma 3. Let $p: M \rightarrow N$ be a submersion between connected (not necessarily Hausdorff ) $C^{1}$-manifolds without boundary, and let $\mathcal{F}$ be the foliation on $M$ induced by p. Let $M$ be equipped with a $C^{1}$-action of a discrete group $G$ such that

$$
p(x \cdot g)=p(x)
$$

for any $x \in M$ and $g \in G$. Then $\mathcal{F}$ is invariant under this action, the equivariant holonomy group of any leaf of $\mathcal{F}$ is trivial, and the action of $G$ is leafwise separated if and only if the orbit spaces of the leaves of $\mathcal{F}$ are Hausdorff. 
If the action of $G$ is leafwise separated and $L$ is a leaf of $\mathcal{F}$ with compact orbit space, then for any $G$-invariant open neighbourhood of $L$ there exists a smaller $G$ invariant open neighbourhood of $L$ which is a union of leaves of $\mathcal{F}$ with compact orbit spaces.

Proof. The first part of the lemma is just a restatement of Example 1 (1).

For the second part, we can assume without loss of generality that $N=\mathbf{R}^{k}$. Let $L$ be a leaf with compact orbit space and $V$ an open $G$-invariant neighbourhood of $L$. Denote by $q: M \rightarrow M / G$ the quotient projection and by $p_{G}: M / G \rightarrow \mathbf{R}^{k}$ the map induced by $p$ as in Example 1 (1). Let $r \in \mathbf{R}^{k}$ be the point with $L \subset p^{-1}(r)$. Since the map $q$ is open and the fiber $p_{G}^{-1}(r)$ is locally connected, we can choose an open neighbourhood $W$ of $q(L)$ such that $W \subset q(V)$ and $W \cap p_{G}^{-1}(r)=q(L)$. By Example $1(1)$ we have $q(L) \cong L / G_{L}$, hence $q(L)$ is compact. Since $M$ is locally compact, so is $M / G$, therefore we can choose a compact neighbourhood $K$ of the compact $q(L)$ in $M / G$ with $K \subset W$.

Now $R=K \backslash \operatorname{Int}(K)$ is compact and $R \cap q(L)=\emptyset$, thus $p_{G}(R)$ is closed and $r \notin p_{G}(R)$. Then $S=p_{G}^{-1}\left(N \backslash p_{G}(R)\right) \cap \operatorname{Int}(K)$ is an open neighbourhood of $q(L)$ and $U=q^{-1}(S) \subset V$ is an open $G$-invariant neighbourhood of $L$.

Let $L^{\prime}$ be a leaf of $\mathcal{F}$ which intersects $U$. Therefore $q\left(L^{\prime}\right) \operatorname{intersects} \operatorname{Int}(K)$ but not $R$. Denote

$$
D=q\left(L^{\prime}\right) \cap \operatorname{Int}(K)=q\left(L^{\prime}\right) \cap K \neq \emptyset .
$$

Observe first that $D$ is open in $q\left(L^{\prime}\right)$. By Example $1(1), q\left(L^{\prime}\right)$ is closed in $M / G$ and therefore $D$ is closed in the compact $K$. Thus $D$ is compact. But since the action is leafwise separated, the space $q\left(L^{\prime}\right) \cong L^{\prime} / G_{L^{\prime}}$ is Hausdorff, so $D$ is closed in $q\left(L^{\prime}\right)$. Finally, since $q\left(L^{\prime}\right)$ is connected, we have $D=q\left(L^{\prime}\right)$, or in other words, $L^{\prime} \subset U$.

Remark. The condition that the action is leafwise separated is a necessary one. For example, take $M=\mathbf{R}^{2} \backslash\{0\}$, and let $p$ be the second projection. Define an action of $\mathbf{Z}$ on $M$ along the fibers of $p$ to be generated by the $C^{1}$-diffeomorphism

$$
\hat{1}(x, y)=\left(x+\frac{\sin (x y)}{2 y}, y\right) \text {. }
$$

The orbit spaces of the leaves in $p^{-1}(0)$ are $S^{1}$, but all the other leaves have noncompact non-Hausdorff orbit spaces.

Proof of Theorem 2. Let $n$ be the dimension of $M$ and $k$ the codimension of $\mathcal{F}$. Denote by $\Gamma^{k}$ the Haefliger $C^{1}$-groupoid [6] of germs of $C^{1}$-diffeomorphisms between open subsets of $\mathbf{R}^{k}$. The space of objects of $\Gamma^{k}$ is $\mathbf{R}^{k}$, the space of morphisms (denoted also by $\Gamma^{k}$ ) is a non-Hausdorff $C^{1}$-manifold and the domain and the codomain maps dom, cod $: \Gamma^{k} \rightarrow \mathbf{R}^{k}$ are local diffeomorphisms. Let $\left(U_{i}, \phi_{i}\right)_{i \in I}$ be the maximal atlas for $\mathcal{F}$. For any $i \in I$ and $g \in G$, the pair $\left(\hat{g}\left(U_{i}\right), \phi_{i} \circ \hat{g}^{-1}\right)$ is also a chart for $\mathcal{F}$, hence there exists a unique $i \cdot g \in I$ such that

$$
\left(\hat{g}\left(U_{i}\right), \phi_{i} \circ \hat{g}^{-1}\right)=\left(U_{i \cdot g}, \phi_{i \cdot g}\right) .
$$

For any $i \in I$ write $\phi_{i}=\left(a_{i}, b_{i}\right): U_{i} \rightarrow \mathbf{R}^{n-k} \oplus \mathbf{R}^{k}$. For any $x \in U_{i} \cap U_{j}$ there exists an open neighbourhood $W \subset U_{i} \cap U_{j}$ of $x$ for which the diffeomorphism $\left.\phi_{i} \circ \phi_{j}^{-1}\right|_{\phi_{j}(W)}$ projects to a diffeomorphism $h_{i, j}: b_{j}(W) \rightarrow b_{i}(W)$. The germ

$$
c_{i, j}(x)=\operatorname{germ}_{b_{j}(x)} h_{i, j} \in \Gamma^{k}
$$


does not depend on the choice of $W$. The maps $b_{i}$ and $c_{i, j}$ form a $\Gamma^{k}$-cocycle on the open cover $\left(U_{i}\right)_{i \in I}$ of $M$ which represents $\mathcal{F}[2,6,8]$.

Let $E=(E, p, w)$ be the principal $\Gamma^{k}$-bundle over $M$ associated to this cocycle $[2,6,8]$. We will give the construction of $E$ explicitly to fix the notation and for convenience of the reader. The space $E$ is the quotient of the subspace

$$
\mathcal{E}=\left\{(\gamma, x, i) \mid \gamma \in \Gamma^{k}, i \in I, x \in U_{i}, \operatorname{dom} \gamma=b_{i}(x)\right\}
$$

of $\Gamma^{k} \times M \times I$ by identifying $(\gamma, x, i)$ with $\left(\gamma \circ c_{i, j}(x), x, j\right)$ for any $\gamma \in \Gamma^{k}, i, j \in I$ and $x \in U_{i} \cap U_{j}$ with $d o m \gamma=b_{i}(x)$. Denote by $[\gamma, x, i]$ the equivalence class of an element $(\gamma, x, i)$ of $\mathcal{E}$. The structure maps $w: E \rightarrow M$ and $p: E \rightarrow \mathbf{R}^{k}$ are given by $w([\gamma, x, i])=x$ and $p([\gamma, x, i])=\operatorname{cod} \gamma$. The left action of $\Gamma^{k}$ on $E$ with respect to $p$ is given by

$$
\gamma^{\prime} \cdot[\gamma, x, i]=\left[\gamma^{\prime} \circ \gamma, x, i\right]
$$

for any $\gamma^{\prime} \in \Gamma^{k}$ and $[\gamma, x, i] \in E$ with $d o m \gamma^{\prime}=p([\gamma, x, i])$. This bundle is principal, so in particular for any $e, e^{\prime} \in E$ with $w(e)=w\left(e^{\prime}\right)$ there exists a unique $\gamma \in \Gamma^{k}$ such that $d o m \gamma=p(e)$ and $\gamma \cdot e=e^{\prime}$. The space $E$ is a $C^{1}$-manifold so that $w$ is a surjective local diffeomorphism and $p$ is a submersion.

The action of $G$ on $M$ lifts to a right $C^{1}$-action of $G$ on $E$ by

$$
[\gamma, x, i] \cdot g=[\gamma, x \cdot g, i \cdot g]
$$

for any $[\gamma, x, i] \in E$ and $g \in G$. Note that $\gamma \cdot(e \cdot g)=(\gamma \cdot e) \cdot g$ and $p(e \cdot g)=p(e)$ for any $\gamma \in \Gamma^{k}, e \in E$ and $g \in G$ with $\operatorname{dom} \gamma=p(e)$. The local diffeomorphism $w$ is equivariant and thus induces a continuous map $w_{G}: E / G \rightarrow M / G$.

Since $p$ is a submersion, it induces a foliation $\mathcal{F}^{\sharp}$ on $E$. Let $L^{\sharp \sharp}$ be a leaf of $\mathcal{F}^{\sharp}$. By construction of $E$ it follows that the set $w\left(L^{\prime \sharp}\right)$ is the underlying set of a leaf $L^{\prime}$ of $\mathcal{F}$, and the restriction $\chi^{\prime}=\left.w\right|_{L^{\prime \sharp}}: L^{\prime \sharp} \rightarrow L^{\prime}$ is a holonomy covering projection. Let $e \in L^{\prime \sharp}$ and $x=\chi^{\prime}(e) \in L^{\prime}$. We can choose $i \in I$ with $x \in U_{i}, \phi_{i}(x)=0$ and $\phi_{i}\left(U_{i}\right)=\mathbf{R}^{k}$. Then $e=[\gamma, x, i]$ for a unique $\gamma \in \Gamma^{k}$. The map $T: \mathbf{R}^{k} \rightarrow U_{i} \subset M$ given by $T(r)=\phi_{i}^{-1}(0, r)$ is a transversal section of $(M, \mathcal{F})$ with $T(0)=x$. Now let $g \in G_{L^{\prime}}$, let $\varphi$ be a path in $L^{\prime}$ from $x$ to $x \cdot g$, and let $\tilde{\varphi}$ be the unique lift of $\varphi$ in $L^{\prime \sharp}$ such that $\tilde{\varphi}(0)=e$. By construction of $E$ it follows that

$$
\tilde{\varphi}(1)=\left[\gamma \circ \operatorname{Hol}_{\hat{g} \circ T, T}^{-1}([\varphi]), x \cdot g, i \cdot g\right]=\left[\gamma \circ \operatorname{Hol}_{\hat{g} \circ T, T}^{-1}([\varphi]), x, i\right] \cdot g,
$$

and therefore, by the definition of the equivariant holonomy homomorphism, we get

$$
\left(\gamma \circ \operatorname{Hol}_{T}^{G}(g,[\varphi]) \circ \gamma^{-1}\right) \cdot \tilde{\varphi}(1)=e \cdot g .
$$

This equation is the key to our result. First we will show that it implies

$$
G_{L^{\prime}}^{\sharp}=G_{L^{\prime \sharp}} \text {. }
$$

If $g \in G_{L^{\prime}}^{\sharp}$, there exists a path $\varphi$ in $L^{\prime}$ from $x$ to $x \cdot g$ such that $\operatorname{Hol}_{T}^{G}(g,[\varphi])=1$. Therefore by the equation (2) we have $e \cdot g \in L^{\prime \sharp}$, so $g \in G_{L^{\prime \sharp}}$. Conversely, if $g \in G_{L^{\prime \sharp}}$ there exists a path $\tilde{\varphi}$ in $L^{\prime \sharp}$ from $e$ to $e \cdot g$ and thus the equation (2) gives $\left(\gamma \circ \operatorname{Hol}_{T}^{G}(g,[w \circ \tilde{\varphi}]) \circ \gamma^{-1}\right) \cdot e=e$. Since $E$ is principal, we have $\operatorname{Hol}_{T}^{G}(g,[w \circ \tilde{\varphi}])=1$ and hence $g \in G_{L^{\prime}}^{\sharp}$.

The equation (2) shows also that the action of $G_{L^{\prime \sharp}}$ on $L^{\prime \sharp}$ is precisely the canonical action of $G_{L^{\prime}}^{\sharp}=G_{L^{\prime \sharp}}$ on the holonomy covering space $L^{\sharp \sharp}$ of $L^{\prime}$. Since 
the equation (3) yields $L^{\prime \sharp} / G_{L^{\prime}}^{\sharp}=L^{\prime \sharp} / G_{L^{\prime \sharp}}$, the assumption that the action of $G$ on $M$ is leafwise separated implies that the action of $G$ on $E$ is leafwise separated.

Now choose $x_{0} \in L$ and $e_{0} \in w^{-1}\left(x_{0}\right)$, and let $L^{\sharp}$ be the leaf of $\mathcal{F}^{\sharp}$ with $e_{0} \in L^{\sharp}$. Hence $w\left(L^{\sharp}\right)$ is the underlying set of $L$ and $\chi=\left.w\right|_{L^{\sharp}}: L^{\sharp} \rightarrow L$ is a holonomy covering projection. As before, choose $i_{0} \in I$ with $x_{0} \in U_{i_{0}}, \phi_{i_{0}}\left(x_{0}\right)=0$ and $\phi_{i_{0}}\left(U_{i_{0}}\right)=\mathbf{R}^{k}$, write $e_{0}=\left[\gamma_{0}, x_{0}, i_{0}\right]$ and define $T: \mathbf{R}^{k} \rightarrow M$ by $T(r)=\phi_{i_{0}}^{-1}(0, r)$. The subspace $L^{\sharp} \cdot G_{L}$ of $E$ is topologically a disjoint union of $\left|G_{L}: G_{L^{\sharp}}\right|$ copies of $L^{\sharp}$. Denote by $\chi \cdot G_{L}: L^{\sharp} \cdot G_{L} \rightarrow L$ the restriction of $w$ on $L^{\sharp} \cdot G_{L}$. The group $G_{L}$ acts on $L^{\sharp} \cdot G_{L}$ and $L$, and the map $\chi \cdot G_{L}$ is an equivariant covering projection. The equation (2) now implies that the left action of the finite group $\gamma_{0} \circ \operatorname{Hol}_{T}^{G}\left(\pi_{1}^{G}\left(L ; x_{0}\right)\right) \circ \gamma_{0}^{-1}$ on $L^{\sharp} \cdot G_{L}$ is transitive along the fibers of the covering projection $\chi \cdot G_{L}$. Since $\chi \cdot G_{L}$ is equivariant, it induces an open surjection

$$
\chi^{G}: L^{\sharp} / G_{L^{\sharp}} \cong L^{\sharp} \cdot G_{L} / G_{L} \longrightarrow L / G_{L} .
$$

The action of $\gamma_{0} \circ \operatorname{Hol}_{T}^{G}\left(\pi_{1}^{G}\left(L ; x_{0}\right)\right) \circ \gamma_{0}^{-1}$ on $L^{\sharp} \cdot G_{L}$ induces an action on $L / G_{L}$ which is transitive along the fibers of $\chi^{G}$. Since the group $\gamma_{0} \circ \operatorname{Hol}_{T}^{G}\left(\pi_{1}^{G}\left(L ; x_{0}\right)\right) \circ \gamma_{0}^{-1}$ is finite and the orbit space $L / G_{L}$ is compact, this implies that the orbit space $L^{\sharp} / G_{L^{\sharp}}$ of the leaf $L^{\sharp}$ of $\mathcal{F}^{\sharp}$ is compact as well.

Let $V$ be an open $G$-invariant neighbourhood of $L$ in $M$. Then $w^{-1}(V)$ is an open $G$-invariant neighbourhood of $L^{\sharp}$ in $E$. We can now apply Lemma 3 to find an open $G$-invariant neighbourhood $U^{\sharp} \subset w^{-1}(V)$ of $L^{\sharp}$ in $E$ which is a union of leaves of $\mathcal{F}^{\sharp}$ with compact orbit spaces. Now $U=w\left(U^{\sharp}\right) \subset V$ is an open $G$-invariant neighbourhood of $L$ in $M$ which is a union of leaves of $\mathcal{F}$ since $w$ maps the leaves of $\mathcal{F}^{\sharp}$ into the leaves of $\mathcal{F}$. If $L^{\prime} \subset U$ is a leaf of $\mathcal{F}$, there is a leaf $L^{\prime \sharp} \subset U^{\sharp}$ of $\mathcal{F}^{\sharp}$ such that $w\left(L^{\prime \sharp}\right)$ is the underlying set of $L^{\prime}$. The restriction $\chi^{\prime}=\left.w\right|_{L^{\prime \sharp}}: L^{\prime \sharp} \rightarrow L^{\prime}$ is equivariant and induces an open surjection

$$
\chi^{\prime G}: L^{\prime \sharp} / G_{L^{\prime \sharp}} \longrightarrow L^{\prime} / G_{L^{\prime}} .
$$

Since any leaf of $\mathcal{F}^{\sharp}$ has compact orbit space, $L^{\prime \sharp} / G_{L^{\prime \sharp}}$ is compact and hence the orbit space $L^{\prime} / G_{L^{\prime}}$ of $L^{\prime}$ is compact as well.

Remark. The proof works also in the $C^{0}$-case. Note that the proof also shows that for any foliation $\mathcal{F}$ of codimension $k$ on $M$ which is invariant under a $C^{1}$-action of a group $G$ on $M$ there exists a (possibly non-Hausdorff) $C^{1}$-manifold $E$ equipped with a $C^{1}$-action $G$ along the fibers of a submersion $p: E \rightarrow \mathbf{R}^{k}$ (i.e. $E$ has a "simple" equivariant foliation of the form described in Lemma 3), and a surjective $G$-equivariant local diffeomorphism $w: E \rightarrow M$ which maps the leaves of $p$ to the leaves of $\mathcal{F}$. Moreover, the map $w$ restricted to a leaf of $p$ is the holonomy cover of the corresponding image leaf of $\mathcal{F}$.

Let us illustrate Theorem 2 on a simple example.

Example 4. Let $M=\mathbf{R} \times S^{1}$, where $S^{1}=\{z \in \mathbf{C}|| z \mid=1\}$, and let $\mathcal{F}$ be the foliation on $M$ given by the submersion $p=p r_{2}: M \rightarrow S^{1}$. Let $G=\mathbf{Z} \oplus \mathbf{Z}$, let $\alpha \in \mathbf{R} \backslash \mathbf{Q}$, and define an action of $G$ on $M$ by

$$
(r, z) \cdot\left(g, g^{\prime}\right)=\left(r+g, \mathrm{e}^{2 \pi i \alpha g^{\prime}} z\right)
$$

for any $(r, z) \in M$ and $\left(g, g^{\prime}\right) \in G$. The foliation $\mathcal{F}$ is invariant under this action. If $L$ is a leaf of $\mathcal{F}$, we have $G_{L} \cong \pi_{1}^{G}(L) \cong \mathbf{Z}$, but the equivariant holonomy group of $L$ is trivial. The group $G_{L}$ acts freely and properly discontinuously on $L$ and 
the orbit space of $L$ is homeomorphic with $S^{1}$, so the action is leafwise separated. Thus the conditions of Theorem 2 are satisfied.

Our second theorem gives an equivariant version of the Thurston generalization [12] of the Reeb stability theorem for foliations of codimension one:

Theorem 5. Let $\mathcal{F}$ be a $C^{1}$-foliation of codimension 1 on a connected $C^{1}$-manifold $M$ without boundary, invariant under a leafwise separated $C^{1}$-action of a discrete group $G$ on $M$. Let $L$ be a leaf of $\mathcal{F}$ with finitely generated equivariant fundamental group such that

(i) the action of $G$ is equivariantly transversely orientable around $L$,

(ii) the orbit space of $L$ is compact, and

(iii) the first equivariant cohomology group of $L$ with coefficients in $\mathbf{R}$ is trivial.

Then the equivariant holonomy group of $L$ is trivial, and for any $G$-saturated open neighbourhood of $L$ there exists a smaller $G$-saturated open neighbourhood of $L$ which is a union of leaves of $\mathcal{F}$ with compact orbit spaces.

Remark. By definition, the first equivariant cohomology group $H_{G_{L}}^{1}(L ; \mathbf{R})$ is the first ordinary cohomology group of the space $L \times_{G_{L}} \mathbf{E} G_{L}$, where $\mathbf{E} G_{L}$ is a universal $G_{L}$-bundle [1]. Since $\pi_{1}^{G}(L) \cong \pi_{1}\left(L \times_{G_{L}} \mathbf{E} G_{L}\right)$, the Hurewicz formula implies that the condition (iii) is equivalent to the condition

$$
\operatorname{Hom}\left(\pi_{1}^{G}(L), \mathbf{R}\right)=\{0\} .
$$

In particular, the exact sequence (1) yields that if the first ordinary cohomology group $H^{1}(L ; \mathbf{R})$ is trivial and $\operatorname{Hom}\left(G_{L}, \mathbf{R}\right)=\{0\}$ then the condition (iii) is satisfied. Next note that if $\pi_{1}(L)$ and $G_{L}$ are finitely generated, then the equivariant fundamental group $\pi_{1}^{G}(L)$ is also finitely generated.

Proof. Choose $x_{0} \in L$ and a transversal section $T: \mathbf{R}^{k} \rightarrow M$ such that $T(0)=x_{0}$. For any $\gamma \in \operatorname{Diff}_{0}(\mathbf{R})$ denote by $d(\gamma) \in \mathbf{R}^{*}=\mathbf{R} \backslash\{0\}$ the differential of $\gamma$ at $0 \in \mathbf{R}$. This gives a homomorphism of groups $d: \operatorname{Diff}_{0}(\mathbf{R}) \rightarrow \mathbf{R}^{*}$. We have the sequence of homomorphisms of groups

$$
\pi_{1}^{G}\left(L ; x_{0}\right) \stackrel{\mathrm{Holl}_{G}^{G}}{\longrightarrow} \operatorname{Diff}_{0}(\mathbf{R}) \stackrel{d}{\longrightarrow} \mathbf{R}^{*} \stackrel{\log |-|}{\longrightarrow} \mathbf{R} .
$$

Now by the assumption (iii) it follows that the composition of these three homomorphisms is trivial, so the assumption (i) implies that also $d \circ \mathrm{Hol}_{T}^{G}$ is trivial. Since $\pi_{1}^{G}\left(L ; x_{0}\right)$ is finitely generated and $\operatorname{Hom}\left(\pi_{1}^{G}\left(L ; x_{0}\right), \mathbf{R}\right)=\{0\}$, the argument of Thurston [12] shows that triviality of $d \circ \mathrm{Hol}_{T}^{G}$ yields that $\mathrm{Hol}_{T}^{G}$ is trivial. We can now apply Theorem 2 .

Example 6. (1) Let $n \geq 2$ and denote by $\mathcal{M}_{n}$ the smooth manifold of $n \times n$ matrices over $\mathbf{R}$. Consider the determinant map det $: \mathcal{M}_{n} \rightarrow \mathbf{R}$. This map is a submersion with connected fibers on the open submanifold of $\mathcal{M}_{n}$

$$
M=\left\{A \in \mathcal{M}_{n} \mid \operatorname{dim} \operatorname{Ker} A \leq 1\right\} .
$$

Hence the fibers of the determinant map are the leaves of a foliation $\mathcal{F}$ on $M$. We will denote by $L_{0}$ the fiber over 0 . We have $M=G L_{n}(\mathbf{R}) \cup L_{0}$, where $G L_{n}(\mathbf{R})$ is open, but not closed in $M$.

Now take $G$ to be the group $S L_{n}(\mathbf{R})$ of matrices in $\mathcal{M}_{n}$ with determinant 1 . The group $G$ acts smoothly on $M$ by

$$
A \cdot g=g^{-1} A, \quad A \in M, g \in G .
$$


This action is along the fibers of the determinant map. In particular,

$$
M / G \cong G L_{n}(\mathbf{R}) / G \cup L_{0} / G \cong \mathbf{R}^{*} \cup L_{0} / G .
$$

The kernel map Ker : $L_{0} \rightarrow \mathbf{R} P_{n-1}$ is a surjective submersion and Ker $A \cdot g=\operatorname{Ker} A$ for any $A \in L_{0}$ and $g \in G$. Therefore Ker factors as

$$
f: L_{0} / G \longrightarrow \mathbf{R} P_{n-1} \text {. }
$$

Since for any $A, B \in L_{0}$ with $\operatorname{Ker} A=\operatorname{Ker} B$ one can find a matrix $g \in G$ such that $g A=B$, the map $f$ is a homeomorphism. In particular, $L_{0} / G$ is compact.

The orbit spaces of the leaves of $\mathcal{F}$ which lie in $G L_{n}(\mathbf{R})$ are the points in $\mathbf{R}^{*}$, and the orbit space of $L_{0}$ is homeomorphic to $\mathbf{R} P_{n-1}$. The space $L_{0} / G$ is not open in $M / G$. This example satisfies the conditions of Lemma 3.

(2) Let $N$ be a connected $C^{1}$-manifold without boundary. A family $\left(f_{\lambda}\right)_{\lambda \in \Lambda}$ of $C^{1}$-diffeomorphisms of $N$ has a compact filling if there exists a compact set $K \subset N$ such that

$$
\bigcup\left\{\left(f_{\lambda_{1}}^{n_{1}} \ldots \circ f_{\lambda_{k}}^{n_{k}}\right)(K) \mid k \geq 1, n_{i} \in \mathbf{Z}, \lambda_{i} \in \Lambda\right\}=N .
$$

Observe that $\left(f_{\lambda}\right)_{\lambda \in \Lambda}$ has a compact filling if and only if the quotient space $N / G_{\left(f_{\lambda}\right)}$ is compact, where $G_{\left(f_{\lambda}\right)}$ is the group of diffeomorphisms of $N$ generated by $\left(f_{\lambda}\right)_{\lambda \in \Lambda}$. The family $\left(f_{\lambda}\right)_{\lambda \in \Lambda}$ is separated if the space of orbits $N / G_{\left(f_{\lambda}\right)}$ is separated.

A $C^{1}$-perturbation of a family $\left(f_{\lambda}\right)_{\lambda \in \Lambda}$ of $C^{1}$-diffeomorphisms of $N$ is a family of $C^{1}$-maps

$$
\left(\Theta_{\lambda}:(-\varepsilon, \varepsilon) \times N \rightarrow N\right)_{\lambda \in \Lambda}
$$

such that $\Theta_{\lambda}^{t}=\Theta_{\lambda}(t,-)$ is a $C^{1}$-diffeomorphism and $\Theta_{\lambda}^{0}=f_{\lambda}$, for any $\lambda \in \Lambda$ and $t \in(-\varepsilon, \varepsilon)$. The perturbation $\left(\Theta_{\lambda}\right)_{\lambda \in \Lambda}$ is separated if the family $\left(\Theta_{\lambda}^{t}\right)_{\lambda \in \Lambda}$ is separated, for any $t \in(-\varepsilon, \varepsilon)$.

Assume that $\left(\Theta_{\lambda}\right)_{\lambda \in \Lambda}$ is a separated $C^{1}$-perturbation of a family $\left(f_{\lambda}\right)_{\lambda \in \Lambda}$ of $C^{1}$-diffeomorphisms of $N$ with a compact filling. Let $M=(-\varepsilon, \varepsilon) \times N$ and let $\mathcal{F}$ be the foliation of codimension one on $M$ given by the submersion $p r_{1}$. For any $\lambda$, we have a $C^{1}$-diffeomorphism $\beta_{\lambda}$ of $M$, given by $\beta_{\lambda}(t, x)=\left(t, \Theta_{\lambda}(t, x)\right)$, which preserves $\mathcal{F}$. Let $G$ be the group of diffeomorphisms of $M$ generated by $\left(\beta_{\lambda}\right)_{\lambda \in \Lambda}$. This action is leafwise separated since the perturbation is separated. Observe that a leaf $L_{t}=p r_{1}^{-1}(t)$ has compact orbit space for the action of $G$ precisely if the family $\left(\Theta_{\lambda}^{t}\right)_{\lambda \in \Lambda}$ has a compact filling. Lemma 3 now implies that there exists $0<\delta<\varepsilon$ such that for any $|t| \leq \delta$, the family

$$
\left(\Theta_{\lambda}^{t}\right)_{\lambda \in \Lambda}
$$

has a compact filling.

\section{ACKNOWLEDGMENT}

I would like to thank Ieke Moerdijk for many discussions that have led up to this paper.

\section{REFERENCES}

[1] M. F. Atiyah, R. Bott, The moment map and equivariant cohomology. Topology 23 (1984), 1-28. MR 85e:58041

[2] R. Bott, Characteristic classes and foliations. Springer Lecture Notes in Math. 279 (1972), 1-94. MR 50:14777

[3] C. Camacho, A. Neto, Geometric Theory of Foliations. Birkhäuser, Boston (1985). MR 87a:57029 
[4] A. Connes, Non-commutative differential geometry. Publ. Math. I.H.E.S. 62 (1985), 41-144. MR 87i: 58162

[5] W. T. van Est, Rapport sur les S-atlas. Astérisque 116 (1984), 235-292. MR 86e:58003

[6] A. Haefliger, Structures feuilletées et cohomologie à valeur dans un faisceau de groupoïdes. Comment. Math. Helv. 32 (1958), 248-329. MR 20:6702

[7] S. Lang, Differential Manifolds. Addison-Wesley, Reading (1972). MR 55:4241

[8] I. Moerdijk, Classifying toposes and foliations. Ann. Inst. Fourier, Grenoble 41, 1 (1991), 189-209. MR 91i:57028

[9] P. Molino, Riemannian Foliations. Birkhäuser, Boston (1988). MR 89b:53054

[10] J. Mrčun, An extension of the Reeb stability theorem. Topology Appl. 70 (1996), 25-55. CMP 96:12

[11] G. Reeb, Sur certaines propriétés topologiques des variétés feuilletées. Actual. Sci. Ind. 1183, Hermann, Paris (1952). MR 14:1113a

[12] W. P. Thurston, A generalization of the Reeb stability theorem. Topology 13 (1974), 347-352.

Mathematical Institute, Utrecht University, P.O. Box 80.010, 3508 TA Utrecht, The NETHERLANDS

Current address: Department of Mathematics, University of Ljubljana, Jadranska 19, 1000 Ljubljana, Slovenia

E-mail address: janez.mrcun@fmf.uni-lj.si 